\title{
Is enough enough?^ What is sufficiency in biometric data?
}

\author{
Galina V. Veres, Mark S.Nixon and John N. Carter \\ Department of Electronics and Computer Science \\ University of Southampton, Southampton, SO17 1BJ
}

\begin{abstract}
Gait recognition has become a popular new biometric in the last decade. Good recognition results have been achieved using different gait techniques on several databases. However, not much attention has been paid to get major questions: how good are biometrics data; how many subjects are needed to cover diversity of population (hypothetical or actual) in gait and how many samples per subject will give good representation of similarities and differences in the gait of the same subject. In this paper we try to answer these questions from the point of view of statistical analysis not only for gait recognition but for other biometrics as well. Though we do not think that we have a whole answer, we content this is the start of the answer.
\end{abstract}

\section{Introduction}

Several biometrics databases were collected in the last decade and many good recognition results have been reported in the literature. However, not much was done to answer the questions will these results be valid for larger dataset? Some studies include a measure of recognition uncertainty, which is a guide to performance on a larger database and can serve to give confidence - the reliability of results. How can these results be used to make conclusions about some population? Is there enough data to get statistically significant results for chosen values of Type I and II error? How does one design a new dataset to get statistically significant results using available benchmark? In this paper we try to find some answers to these questions. Previously several works were published which concerned samples size $[10,9,1,5]$. In this paper we use bounds on the minimum number of samples that guarantees that our future datasets will provide a good estimate of error rate using similar assumptions about distributions of error rates as reported in [5]. We estimate sample size, number of subjects and number of samples per subject assuming that the errors are independently distributed and the binomial law can be approximated by the Normal law. We consider whether some known databases have enough samples to make their results statistically significant. Finally, we looked at how the size of population determines the number of subjects which required to obtain statistically significant results.

The paper is organised as follows. Section 2 presents problem formulation. Formulas for calculating number of samples, number of subjects and number

\footnotetext{
^ He who knows that enough is enough will always have enough. Lao Tzu
} 
of samples per subject are given in Section 3. The design of future datasets is summarized in Section 4. Section 5 verifyes results for some available databases. How population size affects the required number of subjects is presented in Section 6. Finally Section 7 concludes the paper.

\section{Problem formulation}

In biometrics, measurements are abstracted from sensor data. In vision-based biometrics such as face, gait or palm-print recognition, measurements are derived from image data which, say, reflect the topology of target features. When multiple measurements are acquired and stored in a feature vector, each subject can be represented in a mutidimensional space; a 2D measurement space is shown for convenience in Fig. 1. No assumptions have been made about distribution of measurements and graphs are presented for illustration purpose only. Graph a) shows a case when subjects are well separated and graph b) shows more realistic case when feature spaces for different subjects are interconnect. In this space there are a number of subjects for each of whom a number of

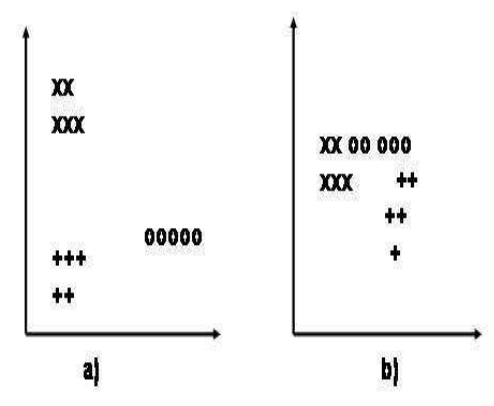

Fig. 1. Possible feature locations for three different subjects $(X,+, O)$ in $2 \mathrm{D}$ case

samples is taken. The complexity of biometric systems, and the need for storage, can make it difficult to acquire multiple samples of biometric data. This is complicated by the covariate structure which concerns factors which influence the measurements or measurement process (and hence recognition capability), but which do not influence the underlying identity of the subject. Should the clusters of measurements, formed by the repeated sampling process, be separate then recognition can be achieved. Recognition performance will suffer when the variance of the clusters (the within-class variance) begins to exceed the variance of the distance between the centres of the clusters (the between-class variance). As such, the number of samples and the number of groups of samples contribute to recognition performance, as do the variances already mentioned. 
The number of subjects/ groups is clearly of interest since biometrics concerns identity, and identity is unique. Consider the introduction of one extra subject: if the new subject falls without the features already recorded then the recognition performance is unlikely to be affected. If on the other hand the extra subject falls within the stored features, then recognition will be influenced by proximity to other subjects. For simplicity, we shall assume that subjects have the same number of samples per subject. The goal of a biometric system is to reduce recognition error by as much as possible for the sample population, whilst leading to a more general conclusion for a larger population. The errors in recognition (the misclassifications by the biometric system) can be represented by a function of the feature vectors obtained for each subject. The dependancy between biometric feature vectors and recognition errors is shown in Fig. 2 where the recognition errors are binary.

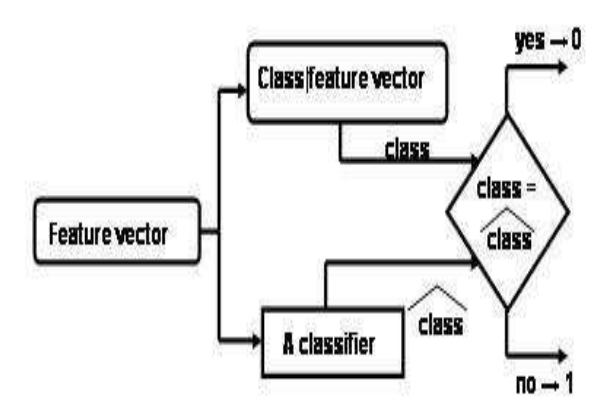

Fig. 2. Recognition process of gait data

Let $X_{i j f}$ will be $f$ th feature for $j$ th repeated samples of $i$ th subject with $f=$ $1, \ldots, N_{f}, N_{f}$ is a number of features, $i=1, \ldots, N_{s}, N_{s}$ is a number of subjects and $j=1, \ldots, n_{g}, n_{g}$ is a number of samples per subject and $n=N_{s} n_{g}$ is a total number of samples per database. Let $Z_{i j}$ be a binary variable and represent the recognition results for $j$ th sample obtained for $i$ th subject, i.e. $Z_{i j}=1$ if there is a recognition error for $j$ th sample of $i$ th subject, and $Z_{i j}=0$ otherwise. $Z_{i j}$ are binomially distributed with probability $p_{i j}$ of drawing 1 and $\left(1-p_{i j}\right)$ of drawing a 0, i.e. $\operatorname{Pr}\left(Z_{i j}=1 \mid X_{i j}\right)=p_{i j}$ and $X_{i j} .=\left[X_{i j 1} X_{i j 2} \ldots X_{i j N_{f}}\right]$. Then dependency between the probability $p_{i j}$ of drawing 1 and feature vectors $X_{i j}$. can be expressed using the logit model. The logistic transformation of the probability of drawing 1 is given by

$$
\operatorname{logit}\left(p_{i j}\right)=\log \left(\frac{p_{i j}}{1-p_{i j}}\right) .
$$

We now treat (1) as a link function in the generalized linear model framework 
and obtained the loglit model

$$
\log \left(\frac{p_{i j}}{1-p_{i j}}\right)=\eta_{i j}=\beta_{i 0}+\beta_{i 1} X_{i j 1}+\ldots+\beta_{i N_{f}} X_{i j N_{f}}=\sum_{f=0}^{f=N_{f}} \beta_{i f} X_{i j f},
$$

where $X_{i j 0}=1$.

Solving for probability $p_{i j}$ :

$$
\hat{p}_{i j}=\frac{\exp \left(\sum_{f=0}^{f=N_{f}} \beta_{i f} X_{i j f}\right)}{1+\exp \left(\sum_{f=0}^{f=N_{f}} \beta_{i f} X_{i j f}\right)} .
$$

For all possible values of $X$ and $\beta$, the logistic transformation ensures that $p$ remains in the $[0,1]$ interval.

The average error rate for an individual $i$ will be calculated as

$$
\hat{p}_{i}=\frac{1}{n_{g}} \sum_{j=1}^{n_{g}} Z_{i j}
$$

where $i=1, \ldots, N_{s}$ and again the dependency between the average error rate for individual $i$ and variable $Z_{i j}$ can be written as a loglit model

$$
\log \left(\frac{p_{i}}{1-p_{i}}\right)=\sum_{j=0}^{j=n_{g}} \beta_{j} Z_{i j} \quad \text { and } \quad \hat{p}_{i}=\frac{\exp \left(\sum_{j=0}^{j=n_{g}} \beta_{j} Z_{i j}\right)}{1+\exp \left(\sum_{j=0}^{j=n_{g}} \beta_{j} Z_{i j}\right)} .
$$

These models can help to predict error rates due to new feature vectors.

The average error rate for a whole data set is computed as

$$
\hat{p}=\frac{1}{N_{s}} \sum_{i=1}^{N_{s}} \hat{p}_{i} .
$$

Under assumption of identical and independently distributed data, recognition errors $Z_{i j}$ can be recognised as Bernoulli trials. Then the total number of errors $s$ in $n=N_{s} * n_{g}$ trials is distributed according to the binomial distribution:

$$
\rho_{n, p}(s)=\left(\begin{array}{c}
n \\
s
\end{array}\right) p^{s}(1-p)^{n-s},
$$

of mean $n p$ and variance $n p(1-p)$. And for a data set size of $n$ samples, the estimate of error probability $p$ is $\hat{p}=\frac{s}{n}$, where $s$ is the number of errors. The expected value of the error rate is $p$ and $\hat{p}$ is the empirical value of error rate estimated on the data set. 
In gait recognition we are interested in confidence intervals. With a certain confidence $(1-\alpha), 0 \leq \alpha \leq 1$, we want the expected value of the error rate $p$ not to exceed a certain value

$$
p<\hat{p}+\epsilon(n, \alpha)
$$

where $\epsilon(n, \alpha)=\beta p$, i.e. $\epsilon(n, \alpha)$ is fixed to a given fraction $\beta$ of $p$. Then the null hypothesis $H_{0}$ is

$$
H_{0}: p-\hat{p}<\beta p
$$

and we want to test it with a confidence of being wrong $\alpha$.

The random variable of which $\hat{p}+\epsilon(n, \alpha)$ is a realisation is a guaranteed estimator of the mean. We are guaranteed, with risk $\alpha$ of being wrong, that the mean does not exceed $\hat{p}+\epsilon(n, \alpha)$ :

$$
\operatorname{Prob}(p \geq \hat{p}+\epsilon(n, \alpha))=\sum_{n p-s \geq \epsilon n} \rho_{n, p}(s) \leq \alpha .
$$

Now we want to find a number of samples $n$, number of subjects $N_{s}$ and a number of samples per subject $n_{g}$ for which (10) holds.

\section{Calculation of subjects number and number of samples per subject for an infinite population}

\subsection{Number of samples in database}

To estimate the total number of samples in the whole database, we will use the Chernoff bound [3], which asserts that with probability $(1-\alpha)$ :

$$
p-\hat{p}<\sqrt{-2 \ln \alpha} \sqrt{\frac{p}{n}}
$$

i.e. $p-\hat{p}<\epsilon(n, \alpha)$ with

$$
\epsilon(n, \alpha)=\sqrt{-2 \ln \alpha} \sqrt{\frac{p}{n}}
$$

A Chernoff bound is a lower bound and allows consideration of tails of distribution. The latter feature is quite important for binomial distribution, since the validity of the approximation of the binomial law by the Normal law in the tail of the distribution is questionable even for large values of the product $n p$.

Assuming that we can fix $\epsilon(n, \alpha)$ to a given fraction $\beta$ of $p$

$$
\epsilon(n, \alpha)=\beta p,
$$

we can assert, with risk $\alpha$ of being wrong that a number of samples

$$
n=\frac{-2 \ln \alpha}{\beta^{2} p}
$$


is sufficient to guarantee that the expected value of error rate $p$ is not worse than $\hat{p} /(1-\beta)$. To use this formula, $p$ needs to be estimated from the results of previous benchmarks.

After recording a new database, it can be desirable to verify the statistical significance of the results. In this case the binomial law is approximated by the Normal law. We will not be able to say with certainty that we have enough samples to represent the population distributed by binomial law, but we will have the certain estimates for ideal case, i.e. normal distribution and it can serve as a guidance for estimation of the sample size.

The best recognizer will obtain an error rate $\hat{p}$ and we would like to test the hypothesis $H_{0}$ :

$$
p-\hat{p}<\beta p
$$

which for small values of $p$ and under the assumption of normal distribution becomes:

$$
p-\hat{p}<z_{\alpha} \sqrt{\frac{p}{n}} .
$$

Solving for $p-\hat{p}$, we obtain

$$
p-\hat{p}<\frac{z_{\alpha}^{2}}{2 n}\left(1+\sqrt{1+\frac{4 n \hat{p}}{z_{\alpha}^{2}}}\right)
$$

Therefore, if we pass the following test:

$$
\frac{z_{\alpha}^{2}}{2 n}\left(1+\sqrt{1+\frac{4 n \hat{p}}{z_{\alpha}^{2}}}\right)<\beta p
$$

we accept $H_{0}$ with risk $\alpha$ of being wrong. Otherwise, the number of examples $n$ is too small to guarantee a relative error bar of $\beta$.

Now we would like to find out the minimum number of subjects (groups) and number of samples per subject needed in the new database.

\subsection{Number of subjects}

We denote by $Z_{. .}=\left(1 / N_{s}\right) \sum_{i=1}^{N_{s}} Z_{i}$. the global mean over $N_{s}$ subjects, where $Z_{i .}=\left(1 / n_{g}\right) \sum_{j=1}^{n_{g}} Z_{i j}$. The expected value of $Z_{i}$. is $p_{i}$ and realizations of $Z_{i}$. are called $\hat{p}_{i}$. The expected value of $Z$.. is $p$ and realizations of it is $\hat{p}$.

We call $\sigma^{2}$ the "between-subject" variance and an estimate of this quantity is given by:

$$
\hat{\sigma}^{2} \simeq \frac{\sum_{i=1}^{N_{s}}\left(\hat{p}_{i}-\hat{p}\right)^{2}}{N_{s}} .
$$


Since $Z_{\text {.. }}$ is the mean over $N_{s}$ subjects of $Z_{i \text {. }}$, its variance is $\sigma^{2} / N_{s}$. Under the assumption that the subject error rates are Normally distributed, the random variable

$$
Z=\frac{p-Z . .}{\sigma / \sqrt{N_{s}}}
$$

obeys the standardized Normal law (with mean 0 and variance 1). Although the approximation of the binomial law by the Normal law is questionable in the tails of distribution it allows to get some estimates for the ideal case and these estimates will serve as a low bound for a real case.

With a risk $\alpha$ of being wrong, we have

$$
p-\hat{p}<z_{\alpha} \frac{\sigma}{\sqrt{N_{s}}},
$$

where $\hat{p}$ is a realization of $Z$.. and $z_{\alpha}$ a threshold obtained from table of the Normal distribution or conveniently approximated by $z_{\alpha} \simeq \sqrt{-\ln \alpha}$.

Then a guaranteed estimator of the average error rate per subject

$$
p-\hat{p}<\epsilon\left(N_{s}, \alpha\right) \text { with } \epsilon\left(N_{s}, \alpha\right)=z_{\alpha} \frac{\sigma}{\sqrt{N_{s}}} .
$$

Remembering (13), we can assert, with risk $\alpha$ of being wrong, that a number of subjects

$$
N_{s}=\left(\frac{z_{\alpha} \sigma}{\beta p}\right)^{2} .
$$

is sufficient to guarantee that the expected value of the average error rate across subjects is not worse than $\hat{p} /(1-\beta)$.

It is worth noticing that $\sigma$ is a function of the number of examples per subject $n_{g}$. However, for large values of $n_{g}$, it is largely independent of $n_{g}$. Since $\sqrt{p / n_{g}}$ is a lower bound of $\sigma$, the hypothesis that $\sigma$ is largely independent of $n_{g}$ will be verified when $n_{g} \gg 1 / p$.

\subsection{Number of samples per subject}

Now the problem of determining the number of samples per subject is addressed. We try to find a balance between "within-subject" and "between-subject" variance. The following assumption is made that the empirical subject error rates are random variables $Z_{i}$, normally distributed with mean $p_{i}$ and variance $\sigma^{2}$, where $\sigma^{2}$ is "between-subject" variance, an estimate of which is given by (19).

The number of examples per subject $n_{g}$ can be expressed as a function of the ration $\gamma$ of the "between-subject" variance $\sigma^{2}$ and the "within-subject" variance $w^{2}$, which can be estimated from a benchmark data or some assumption can be made about it. Then

$$
\hat{\gamma}=\frac{\sigma^{2}}{w^{2}}
$$

$\gamma$ cannot be less than 1 by definition, therefore $\gamma \simeq \max (1, \hat{\gamma})$. Then total number of samples is $n^{\prime}=\gamma n$, where $n$ is calculated as (14). 
At the same time a total number of samples $n^{\prime}=N_{s} n_{g}$, then the number of samples per subject can be calculated as

$$
n_{g}=\frac{\gamma n}{N_{s}}
$$

\section{Designing database sizes for future acquisition}

We need to find out how many samples, subjects and samples per subject are needed for gait recognition to get statistically significant results. Gait recognition aims to discriminate individuals by the way they walk and has the advantage of being non-invasive, hard to conceal, being readily captured without a walker's attention. It is less likely to be obscured than other biometric features. The large database (LDB) described in [8] is used as benchmark for calculating sample sizes for the new database. LDB consists of 115 subjects performing a normal walk and can help to determine which image information remains unchanged for a subject in normal conditions and which changes significantly from subject to subject, i.e. it represents subject-dependent covariates. We used our own database as benchmark for convience, since we know its structure and good recognition results were reported using this database [11]. It is assumed that a new database will be recorded in similar or near conditions to the recording of the benchmark and the quality of images in the new database will be not much worse than in the benchmark. These assumptions are quite realistic and ready to achieve. With confidence of $95 \%(\alpha=0.05)$, we want the expected value of error rate $p$ to be not worse than 1.25 times the error rate of the best recognizer $1.25 \hat{p}$, i.e. $\beta=0.2, z_{\alpha}=1.65$. We will calculate samples size for the expected values of error rates $0.01,0.02$ and 0.03 , since many recognition papers reported the correct classification rate around or above $97 \%$; and $\hat{p}=0.0153$ which was estimated from LDB for the best classifier. Then the choice of samples size will be trade off between the expected error rate and time and recourses needed to record the new database. The following values were estimated using LDB: "between-subject" variance $\hat{\sigma}^{2}=0.0019$ and $\gamma \simeq 3$.

Then using formula (14), the number of samples $n$ in new database should be as in Table 1, i.e. to guarantee correct classification rate (CCR) of $99 \%$ we need almost 15000 samples in the database, practically 7500 samples are needed for CCR of $98 \%$ and lastly 5000 samples are needed for CCR of $97 \%$. The numbers

Table 1. Number of samples, subjects and number of samples per subject needed

\begin{tabular}{|c|c|c|c|}
\hline$p$ & 0.01 & 0.02 & 0.03 \\
\hline$n$ & 14975 & 7490 & 5000 \\
\hline$N_{s}$ & 1294 & 324 & 144 \\
\hline$n_{g}$ & 35 & 70 & 105 \\
\hline
\end{tabular}

of subjects $N_{s}$ from formula (23) are presented as well in Table 1, i.e. this number 
of subjects will be sufficient to guarantee that the expected error rate across all subjects is not worse than $1.25 \hat{p}$. Approximately 1300 subjects are needed to guarantee $99 \%$ CCR, 324 subject for $98 \%$ CCR and 144 subjects for $97 \%$ CCR. Since a number of subjects was obtained by approximation of the binomial law, it is better to take more subjects than the numbers reported if resourses and time are available for collecting bigger data sets. Finally, using formula (25) and results presented in Table 1 so far, we can calculate the number of samples per subject $n_{g}$. The results are presented in Table 1 . The number of samples needed per subject is 35, 70 and 105 for $99 \%, 98 \%$ and $97 \%$ CCRs recpectively. When collecting 70 or 105 samples per subject, extra attention should be paid to the design of the experiment. Since walking for too long (around one hour for 70 samples) will make a person tired and the tiredness will affect their gait. Therefore it is advisable to divide recording to several slots and record the person during a couple of days. As it was reported previously gait is not affected by time when only days pass between recording sections [13].

\section{$5 \quad$ Verifying the statistical significance of the results for available databases}

Several databases were recorded recently describing different biometrics such as gait, face, iris, finger print. We would like to look at some of the them from the point of the view of statistical significants of the results using formula (18). The databases considered are LDB describing gait from the University of Southampton mentioned above [12], CASIA iris image database [4, 2], FERET database of facial images [6], subset of BEN database of fingerprint images [14] and FRVT2002 database of faces [7]. CCRs reported for these databases and a number of samples used to obtain these CCRs are presented in Table 2. All these

Table 2. Characteristics of databases

\begin{tabular}{|c|c|c|c|c|c|}
\hline database & LDB & BEN & FRVT2002 & FERET & CASIA \\
\hline$\hat{p}$ & 0.0153 & 0.0103 & 0.27 & 0.03 & 0.0086 \\
\hline $\mathrm{n}$ & 2161 & 12000 & 121589 & 2392 & 756 \\
\hline
\end{tabular}

databases have a good number of samples collected in the similar conditions and CCRs around or above $97 \%$ were reported for all of them except FRVT2002, where CCR is $73 \%$. We included FRVT2002 in our investigation since it has the highest number of samples $n=121589$. It would be interesting to see the results of applying formula (18) to such a big dataset.

Using the data from Table 2 the left-hand side of the formula (18) is calculated for each dataset with $\alpha=0.05$ and results are presented Table 3. For $\beta=0.2$ the right-hand side of the formula (18) gives $0.002,0.004$ and 0.006 for $p=0.01,0.02$ and 0.03 respectively. It can be seen from Table 5 , that only BEN 
Table 3. Verification of the statistical significance of the results

\begin{tabular}{|c|c|c|c|c|}
\hline LDB & BEN & FRVT2002 & FERET & CASIA \\
\hline 0.0051 & 0.0016 & 0.0025 & 0.0064 & 0.0076 \\
\hline
\end{tabular}

database has enough samples for all expected values of the error rates to guarantee a relative error bar of $\beta$. FERET and CASIA do not have enough samples for any chosen expected values of the error rates to guarantee a relative error bar of $\beta$. LDB and FRVT2002 have enough samples for some expected values of the error rate. All this means that either the number of samples in data bases has to be increased or if it is not possible the relative error bar of $\beta$ should be increase if it is possible for a given applications.

\section{Database size for a population of finite size}

The number of subjects $N_{s}$ obtained in Table 1 was calculated without taking into consideration the size of the population. When the population is large enough in comparison to the sample size or it is desirable later to extend analysis of data for larger population, then the number of subjects in Table 1 is valid. However, when population is comparibale in size with the sample size, the finite population correction factor is needed, i.e. the final number of subjects $n_{f}$ will be

$$
n_{f}=\frac{N_{s}}{1+\frac{N_{s}}{N}},
$$

where $N$ is a population size, $N_{s}$ is a sample size. These statements are made under assumption that the population is sampled uniformly. When it is not possible to sample uniformly from the population bigger sample sizes will be meeded.

Biometrics can be used both on a large population and on a relatively small one. For instance, gait recognition can be used to make identification and statistical conclusions for population of Britain (58 million people) or to gain a security access to the firm of 10000 people. The question arises how the size of the population will affect the number of subjects needed for investigation? To get some approximate knowledge about final size, we look at the concrete example from Table 1 , when number of subjects $N_{s}=324$ for $p=0.02$. The dependency between size of population $N$ and final number of subject $n_{f}$ was calculated, where $N$ will change from 500 to 15 million people by 100 people. The calculations showed that after some size of population the number of subjects needed to characterise this population stays practically the same. In this case, from size of population $N$ equals around 10.5 million people, the corrected number of subjects stays at the same level of 323.9 subjects, thus $n_{f}=324$, and we got the final number of subjects 323.007 or $n_{f}=324$ for a population size from 104400. This means that if we are dealing with the population size of $N=104400$ people, sample of 324 people will be enough to draw statistically 
significant conclusions about this population and the finite population correction factor does not affect the results. So far we looked at what happens when the population is quite large. However, it is interesting how the number of subjects needed will change when the size of population is comparable to the sample size (here number of subjects). Fig. 3 shows a dependency between population size and the final number of subjects needed when the former is comparable in size with the later, i.e. in this case $N=15000$ or below. In this case the finite

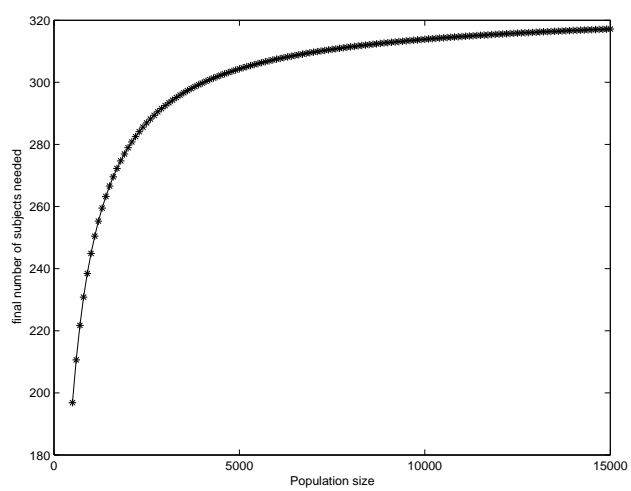

Fig. 3. Dependency between population size and the number of subjects for smaller populations

population correction factor helps to reduce the small size noticeably for the population of 3000 people or below. After that the growth in the sample size is very slow and increase is from 293 subjects for the population of 3000 people to 317 subjects for the population of 15000 people. All these conclusions are valid when the population was uniformly sampled.

\section{Conclusions}

In this paper we tried to answer the question how much biometrics data needed from the point of view of the error rates of the best classifier and statistical significance of the results. The logit model of dependency between recognition errors and feature vectors is described, since binomial variables (error rates) are dependent on continuous variables (feature vectors). The estimates for sample size, number of subjects and a number of samples per subjects are given for several expected error rates and Type I error of 0.05 and power 0.8 using bounds for binomial distribution when it is possible or using approximation of the binomial law by the Normal law in other cases. Several existing databases were checked whether they have enough samples to obtain statistically significant results. Lastly it was shown how the number of subjects required changes for different sizes of population, and it is interesting that it appears to be largely independetnt of the population, when population size is bigger than sample size. 


\section{Acknowledgment}

We gratefully acknowledge partial support by the Defence Technology Centre $8-10$ supported by General Dynamics.

\section{References}

1. J.F. Bromahgin Sample Size Determination for Interval Estimation of Multinomial Probabilities The American Statistician, vol. 47, 203-206, 1993

2. CASIA iris database. Institute of Automation, Chinese Acadamy of Sciences.http://www.sinobiometrics.com/casiairis.htm.

3. H. Chernoff A Measure of Asymptotic Efficiency for Tests of a Hypothesis Based on the Sums of Observations Annals of Mathematical Statistics, vol. 23, 493-509, 1952

4. C. T. Chu and C. Chen High Performance of Iris Recognition Based on LDA and LPCC Proc. 17th IEEE International Conf. on Tools with Artificial Intelligence, Hong Kong, 2005

5. I. Guyon, J. Makhoul, R. Schwartz and V. Vapkin What Size Test Set Gives Good Error Rates Estimates? IEEE Trans. Pattern Analysis and Machine Intelligence, vol. 20 (1), 52-64, 1998

6. P.J. Phillips, H. Moon, S.A. Rizvi and P.J. Rauss The FERET Evaluation Methodology for Face-Recognition Algorithms IEEE Trans. Pattern Analysis and Machine Intelligence, vol. 22 (10), 1090-1104, 2000

7. P.J. Phillips, P. Grother, R. Micheals, D.M. Blackburn, E. Tabassi and M. Bone Face Recognition Vendor Test 2002: Overview and Summary ftp://sequoyah.nist.gov/pub/nist_internal_reports/ir_6965/FRVT_2002_ Overview_and_Summary.pdf, March 2003

8. J. D. Shutler, M. G. Grant, M. S. Nixon, and J. N. Carter On a Large SequenceBased Human Gait Database Proc. 4th International Conf. on Recent Advances in Soft Computing, Nottingham (UK), 2002

9. S.K. Thompson Sample Size for Estimating Multinomial Proportions The American Statistician, vol. 41, 42-46, 1987

10. R.D. Tortora A Note on Sample Size Estimation for Multinomial Populations The American Statistician, vol. 32, 100-102, 1978

11. G.V. Veres, L. Gordon, J.N. Carter and M.S. Nixon What image information is important in silhouette-based gait recognition? Proceedings of IEEE Computer Society Conference on Computer Vision and Pattern Recognition, Washington, D.C., USA, II: 776-782 2004.

12. G.V. Veres, M.S. Nixon and J.N. Carter. Model-based approaches for predicting gait changes over time Proc. of International Workshop on Pattern Recognition, Beijing (China), 2005.

13. G.V. Veres, M.S. Nixon and J.N. Carter Modelling the time-variant covariates for gait recognition accepted to 5th International conference on Audio- and VideoBased Viometric Person Authentication, New York, USA, 2005.

14. C. Wilson, R.A. Korves, B. Ulery. M. Zoepfl, M. Bone, P. Grother, R. Micheals, S. Otto and C. Watson Fingerprint Vendor Technology Evaluation 2003: Summary of Results and Analysis Report NISTIR 7123, June 2004, http://fpvte.nist.gov/report/ir_7123_analysis.pdf

This article was processed using the $\mathrm{AT}_{\mathrm{E} X} \mathrm{X}$ macro package with LLNCS style 